\title{
Highly Sensitive Hydrogen Sensor Based on Palladium-Coated Tapered Optical Fiber at Room Temperature ${ }^{+}$
}

\author{
Mohammed Majeed Alkhabet 1, Saad Girei 1,2, Suriati Paiman 3 , Norhana Arsad 4, \\ Mohd Adzir Mahdi ${ }^{1}$ and Mohd Hanif Yaacob 1,* \\ 1 Wireless and Photonics Network Research Center, Faculty of Engineering, University Putra Malaysia, \\ UPM Serdang 43000, Selangor, Malaysia \\ 2 Department of Computer Engineering, Federal Polytechnic Mubi, Adamawa State, Nigeria \\ 3 Department of Physical, Faculty of Science, University Putra Malaysia, Serdang 43000, Selangor \\ 4 Department of Electrical, Electronic and System Engineering, Faculty of Engineering and Built \\ Environment, University Kebangsaan Malaysia, UKM Bangi 43600, Selangor, Malaysia \\ * Correspondence: hanif@upm.edu.my; Tel.: +60-16-328-9044 \\ + Presented at the 7th International Electronic Conference on Sensors and Applications, 15-30 November \\ 2020; Available online: https://ecsa-7.sciforum.net/.
}

Published: 15 November 2020

\begin{abstract}
This paper describes the application of a palladium (Pd)-coated tapered optical fiber in order to develop hydrogen $\left(\mathrm{H}_{2}\right)$ sensor. A transducing channel was fabricated with multimode optical fiber (MMF) with cladding and core diameters of $125 \mu \mathrm{m}$ and $62.5 \mu \mathrm{m}$, respectively, to enhance the evanescent field of light propagation through the fiber. The multimode optical fiber was tapered from a cladding diameter of $125 \mu \mathrm{m}$ to a waist diameter of $20 \mu \mathrm{m}$, waist-length of 10 $\mathrm{mm}$, and down taper and up of $5 \mathrm{~mm}$ and coated with Pd using the drop-casting technique. In order to establish the palladium's properties, various characterization techniques were applied, such as FESEM, EDX, and XRD. The developed palladium sensor functioned reproducibly at a gas concentration of $0.125 \%$ to $1.00 \% \mathrm{H}_{2}$ at room temperature in the synthetic air. In this case, the response and recovery times were 50 and $200 \mathrm{~s}$, respectively. Furthermore, this study demonstrated that the production of a dependable, effective, and reproducible $\mathrm{H}_{2}$ sensor by applying a basic, costeffective method is possible.
\end{abstract}

Keywords: tapered optical fiber; hydrogen $\left(\mathrm{H}_{2}\right)$; palladium (Pd); drop-casting technique

\section{Introduction}

Hydrogen $\left(\mathrm{H}_{2}\right)$ has high energy content, making it an ideal clean fuel with several application potentials in different industries [1]. The combustion of hydrogen in the air creates water, a common earth element; $\mathrm{H}_{2}$ can hence be sourced from water, hydrocarbons, and biomass. $\mathrm{H}_{2}$, a clean source of energy, can be utilized to fulfil the ever-increasing demand for energy. However, hydrogen is flammable at concentrations $>4 \mathrm{vol} \%$ in the air and can explode at a wider range of $15-59 \mathrm{vol} \%$ at standard pressure [2]. Therefore, effective $\mathrm{H}_{2}$ monitoring systems must be developed to help identify leakages arising from the invisible, flammable, and odourless nature of the gas.

On the other hand, optical sensors rely on the use of optical fibers that offer interesting properties, such as lightweight, small size, resistance to electromagnetic interference, instability, and rigidity in harsh environments [3]. These interesting properties make optical fibers ideal candidates for sensing in a rugged environment [4]. Palladium $(\mathrm{Pd})$ is currently receiving interest in many applications, such as $\mathrm{H}_{2}$, the hydrogenation process, and the detection of $\mathrm{H}_{2}$. The high sensitivity, 
good selectivity, and ability to operate at room temperature of Pd nanoparticles make it the ideal and noble metallic catalyst for the $\mathrm{H}_{2}$ sensing [5].

In recent years, several hydrogen optical sensors have been reported using palladium as an energy transformer. Most of them rely on fiber gratings (FBGs) [6] and plastic optical fibers $[7,8]$. Most fiber-optic sensors need some modification to the cladding to make it sensitive to its surroundings [9]. These modifications include chemical etching [10], side polishing [11], or shape-D [12].In this research, dissolved tapered optical fiber-coated palladium is used to detect gaseous hydrogen.

\section{Experiments}

\subsection{Fabrication of Tapered Optical Fiber}

The $\mathrm{H}_{2}$ gas sensor was fabricated using Multimode Optical fiber (MMF) with cladding and core diameters of $125 \mu \mathrm{m}$ and $62.5 \mu \mathrm{m}$, respectively, as a transducing platform. Tapered MMF was used as the transducing platform. The tapering was done using the Vytran glass processing machine (Vytran GPX-3400). The machine works based on the heating and pulling process, using a graphite filament as a heater to achieve the desired geometry of the tapered profile. The MMF was tapered from a cladding diameter of $125 \mu \mathrm{m}$ to a waist diameter of $20 \mu \mathrm{m}$, waist-length of $10 \mathrm{~mm}$, and down taper and up of $5 \mathrm{~mm}$. Figure 1 shows the image of the fabricated tapered optical fiber showing the down taper region. This tapered geometry, as per [13], offers a strong response between the gassensing layer and the evanescent field.

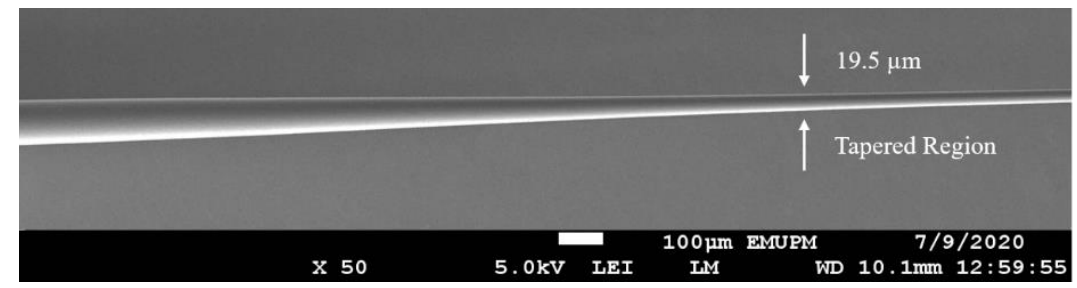

Figure 1. SEM micrograph of the transition region of the prepared tapered MMF.

\subsection{Palladium Functionalization of the Tapered Optical Fiber}

The Pd sensor was fabricated following a simple one-step process. First, $0.1 \mathrm{~mL}$ of hydrochloride acid was mixed with $0.9 \mathrm{~mL}$ of palladium chloride $\left(\mathrm{PdCl}_{2}\right)$, followed by the addition of $10 \mathrm{~mL}$ of deionized water. The solution was placed in an ultrasonic bath and left for $15 \mathrm{~min}$ to homogenize. The coating of the tapered optical fiber was done using the drop-casting technique. A drop of the mixture (approx. $10 \mu \mathrm{L}$ ) was dropped into the base of the tapered optical fiber through a micropipette and heated at $80^{\circ} \mathrm{C}$ for $15 \mathrm{~min}$ in the oven to ensure complete evaporation of the aqueous medium [14].

The experimental setup of the gas optical sensing system consists of a light source (Tungsten Halogen, HL-2000, Ocean Optics USA) with coverage wavelength of 360 to $2500 \mathrm{~nm}$, a spectrophotometer (USB 4000, Ocean Optics USA) with a detection range of 200-1100 for monitoring the optical absorption spectrum, and a dedicated gas chamber. The Pd coated sensor was placed in a closed gas unit and purged with the centrifuge from a computer-regulated mass flow controller at a gas flow rate of $200 \mathrm{sccm}$. Figure 4 illustrates the experimental setup of the $\mathrm{H}_{2}$ sensor. 


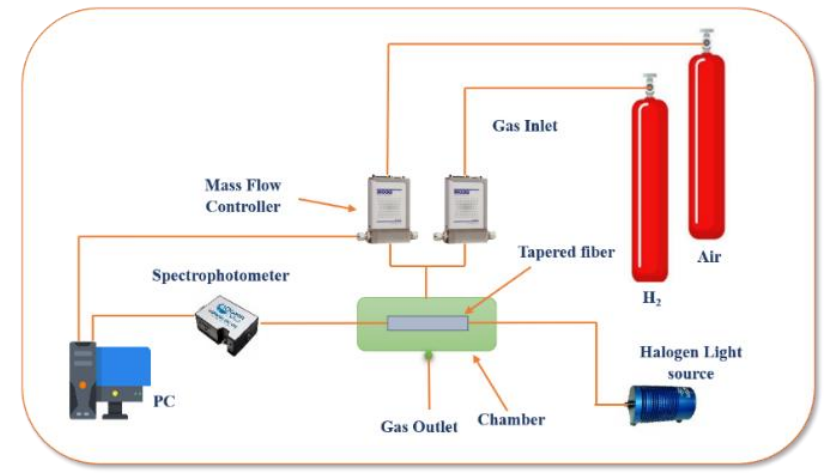

Figure 2. Illustrates the experimental setup of the $\mathrm{H}_{2}$ sensor.

\subsection{Material Characterization}

The films' morphology was observed using FEESEM (JSM-7600F), while their elemental composition was determined through an EDX analysis. Material identification, crystallinity, and phase transition of Pd were observed by an XRD analysis (APD 2000). Figure 3 illustrates the FESEM images of the Pd nanoparticles. Pd NPs are clearly formed and separated.
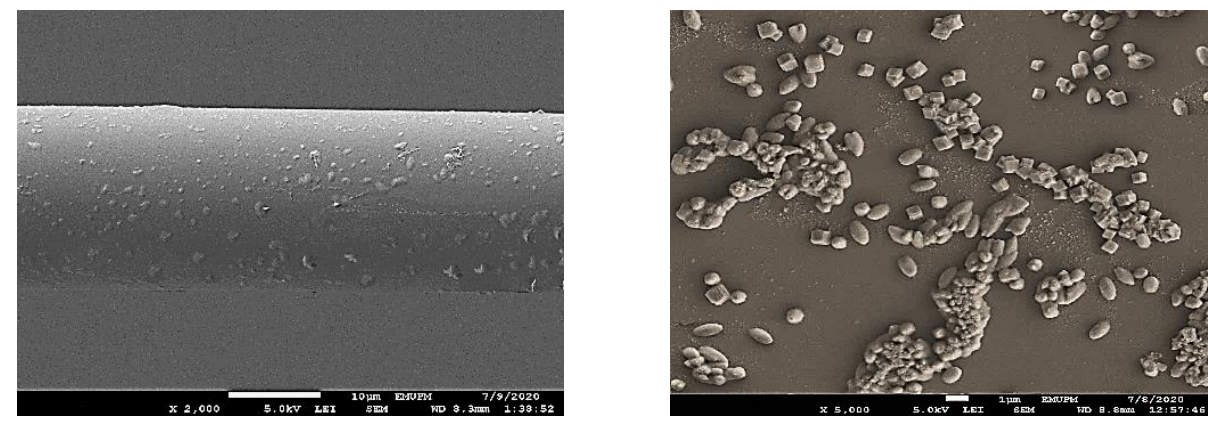

Figure 3. The FESEM micrograph of Pd NPs.

The EDX pattern of Pd shown in Figure 4a revealed that the important elements in Pd films are $\mathrm{Pd}$ and $\mathrm{O}$, as evidenced by their respective peaks. Figure $4 \mathrm{~b}$ reveals XRD patterns of the Pd-coated sensor recorded in range $2 \theta$, from $30^{\circ}$ to $90^{\circ}$. There are five distinct reflections in the reflection at $40.02^{\circ}(111), 46.49^{\circ}(200), 68.05^{\circ}(220), 82.74^{\circ}(311)$ and $86.27^{\circ}(222)$. These characteristic reflections can be categorized into a face-centric cubic structure (fcc) of Pd. The stronger reflection (111) compared to the other four may indicate the preferred growth direction of the nanocrystals [15].

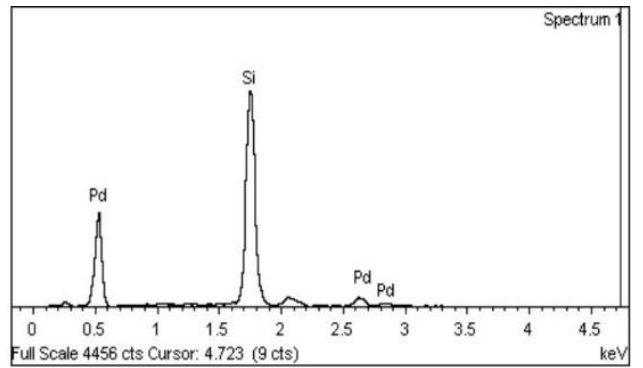

(a)

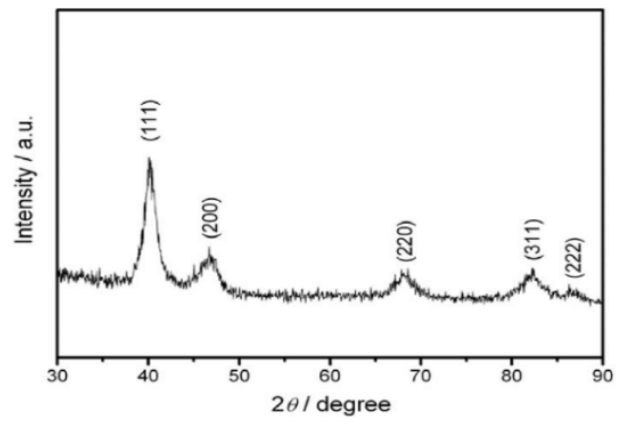

(b)

Figure 4. (a) EDX measurement of Pd NPs, and (b) XRD pattern of Pd NPs. 


\section{Results and Discussion}

Figure 5 depicts the absorption spectra of the sensor coated with Pd to synthetic air and $1.00 \%$ $\mathrm{H}_{2}$ at room temperature. The Pd sensor demonstrated notable changes in absorbance, especially in the wavelength range of $550-850 \mathrm{~nm}$, as shown in Figure 5a. The overall sensor performance of the $\mathrm{Pd}$ coated sensor was monitored in terms of cumulative absorption, which is the product of a combination of response curves over a particular wavelength range. Figure $5 \mathrm{~b}$ displays the dynamic response of the $\mathrm{Pd}$ coated sensors of about $0.125 \%$ to $1.00 \% \mathrm{H}_{2}$ concentrations in air, at room temperature. The response time and recovery time of the Pd costed sensor was $50 \mathrm{~s}$ and $200 \mathrm{~s}$, respectively. Changes in absorption at $0.125 \% \mathrm{H}_{2}$ are about $24 \%$ and $52 \%$ higher at $1.00 \% \mathrm{H}_{2}$. The $\mathrm{Pd}$ coated sensor showed stronger absorbance and recovery of $\mathrm{H}_{2}$ at higher absorption changes as compared to the works of $[8,16]$. Sensor repeatability was confirmed by exposure of the sensor to 3 cycles of $1.00 \% \mathrm{H}_{2}$, as shown in Figure 5c. Overall, the Pd coated sensor showed a high level of absorption and good repeatability of $\mathrm{H}_{2}$.

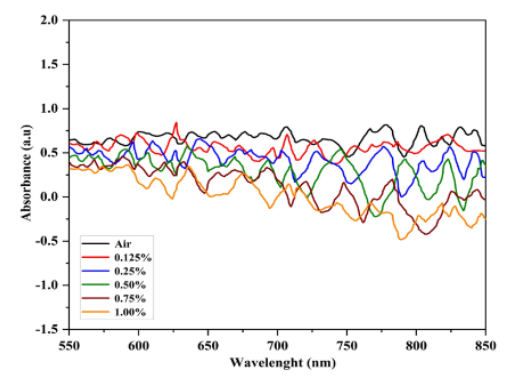

(a)

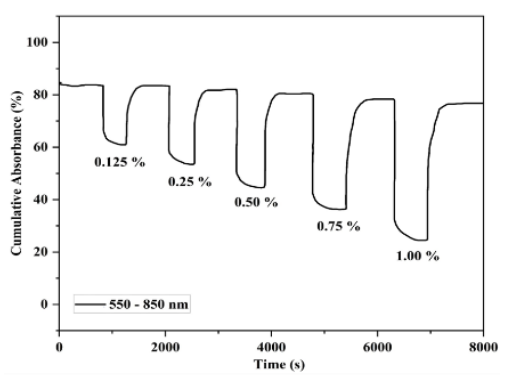

(b)

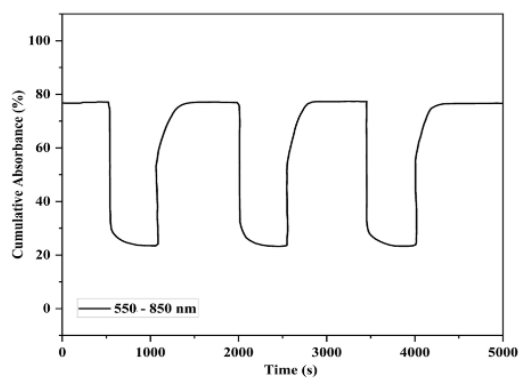

(c)

Figure 5. (a) Absorbance versus optical wavelength, (b) Dynamic absorbance curves, and (c) repeatability of Pd coated sensor.

Absorbance versus $\mathrm{H}_{2}$ concentration for Pd coated sensors is shown in Figure 6a. The sensitivity obtained from Pd coated sensors was $15.40 \mathrm{vol} \%$, with a slope of linearity of $89 \%$. A test for selectivity was also done for the Pd coated sensor on tapered optical fiber toward $\mathrm{NH}_{3}$ and $\mathrm{CH}_{4}$ gas at $1.00 \%$ concentration, as shown in Figure $6 \mathrm{~b}$. The Pd coated sensor showed a remarkably high $\mathrm{H}_{2}$ absorbance response with a weak response for other gases. According to [17], $\mathrm{CH}_{4}$ gas is a stable gas that requires very high energy to dissociate $\mathrm{H}$ from $\mathrm{C}$; hence, a high operating temperature is needed to enhance sensitivity toward this gas. The sensor is less sensitive toward $\mathrm{NH}_{3}$, probably because of $\mathrm{Pd}$ since it is more suitable for dissociating the $\mathrm{H}_{2}$ gas [18].

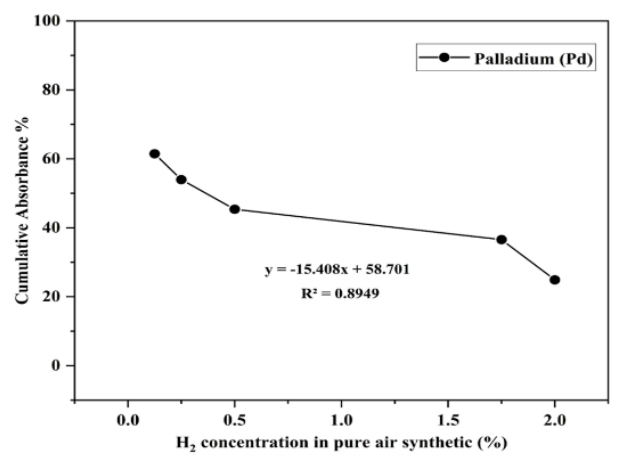

(a)

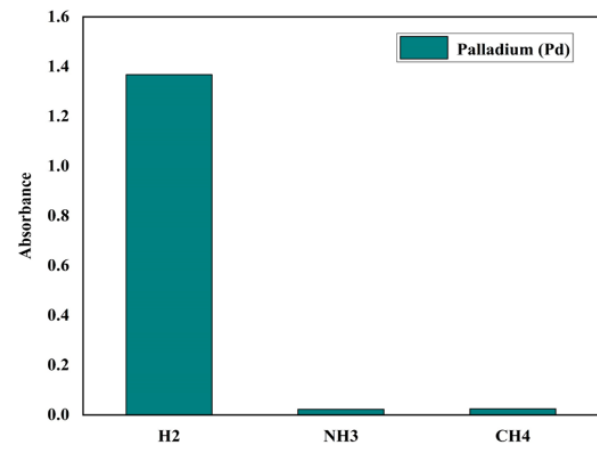

(b)

Figure 6. (a) Absorbance changes at different $\mathrm{H}_{2}$ concentrations for Pd coated sensors, (b) The selectivity of Pd coated sensor. 


\section{The Sensing Mechanism for Tapered Pd NPs Coated Optical Fibers}

The Pd-coated fiber sensor's optical response occurs because of the reaction of palladium to hydrogen gas, as shown in Figure 7. Pd absorbs $\mathrm{H}_{2}$ gas molecules, resulting in it changing into PdHx (where a small percentage expands the Pd particle size), and its functions are lesser than pure Pd. Following this, the hydrogen molecule splits into single hydrogen atoms at a dissociation rate, which is highly efficient. Subsequently, the Pd layer increases in thickness and size while absorbing hydrogen, thereby also changing the layer's optical properties. The real and imaginary parts alter the permittivity of the Pd layer to result in a corresponding change of boundary conditions on the sensor surface.

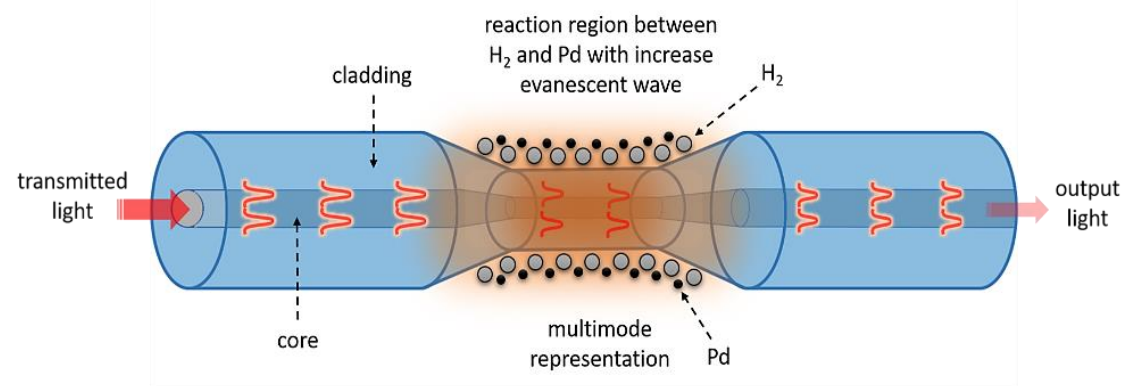

Figure 7. Hydrogen-Palladium sensing mechanism.

\section{Conclusions}

This study demonstrated that optical fiber sensors could be developed from Pd NPs by employing a drop-casting technique. The performance of the developed sensor was evaluated in terms of its response at room temperature using different concentrations of $\mathrm{H}_{2}$ gas. These evaluations indicated that the Pd-coated sensor exhibited a 52\% change in the absorbance response when exposed to $1.00 \% \mathrm{H}_{2}$ in synthetic air. The outcome of the study suggests that it is possible to develop an efficient, reliable, and reproducible $\mathrm{H}_{2}$ sensor by using a cost-effective and straightforward approach under real atmospheric conditions.

Author Contributions: Conceptualization, M.M.A., S.G. and M.H.Y.; methodology, M.M.A. and M.H.Y.; writing-original draft preparation, M.M.A.; Review and editing, M.H.Y., S.G., M.A.M., S.P. and N.A.; All authors have read and agreed to the published version of the manuscript.

Funding: University Putra Malaysia funded this research, grant number GP-IPS/2019/9674900.

Conflicts of Interest: The authors declare no conflict of interest.

\section{References}

1. Parra, D.; Valverde, L.; Pino, F.J.; Patel, M.K. A review on the role, cost and value of hydrogen energy systems for deep decarbonisation. Renew. Sustain. Energy Rev. 2019, 101, 279-294.

2. Fisser, M.; Badcock, R.A.; Teal, P.D.; Hunze, A. Optimizing the sensitivity of palladium based hydrogen sensors. Sens. Actuators B Chem. 2018, 259, 10-19, doi:10.1016/j.snb.2017.11.180.

3. Pospíšilová, M.; Kuncová, G.; Trögl, J. Fiber-optic chemical sensors and fiber-optic bio-sensors. Sensors 2015, 15, 25208-25259.

4. Rajan, G. Optical Fiber Sensors: Advanced Techniques and Applications; CRC Press: Boca Raton, FL, USA, 2015; ISBN 1482228297.

5. Chen, A.; Ostrom, C. Palladium-based nanomaterials: Synthesis and electrochemical applications. Chem. Rev. 2015, 115, 11999-12044.

6. Dai, J.; Yang, M.; Yu, X.; Lu, H. Optical hydrogen sensor based on etched fiber Bragg grating sputtered with Pd/Ag composite film. Opt. Fiber Technol. 2013, 19, 26-30, doi:10.1016/j.yofte.2012.09.006.

7. Arasu, P.T.; Noor, A.S.M.; Khalaf, A.L.; Yaacob, M.H. Highly sensitive plastic optical fiber with palladium sensing layer for detection of hydrogen gas. In Proceedings of the 2016 IEEE Region 10 Symposium (TENSYMP), Bali, Indonesia, 9-11 May 2016; pp. 390-393. 
8. Arasu, P.T.; Noor, A.S.M.; Khalaf, A.L.; Yaacob, M.H. Highly sensitive plastic optical fiber with palladium sensing layer for detection of hydrogen gas. In Proceedings of the 2016 IEEE Region 10 Symposium (TENSYMP), Bali, Indonesia, 9-11 May 2016; pp. 390-393, doi:10.1109/TENCONSpring.2016.7519438.

9. Kim, Y.H.; Kim, M.J.; Rho, B.S.; Park, M.-S.; Jang, J.-H.; Lee, B.H. Ultra sensitive fiber-optic hydrogen sensor based on high order cladding mode. IEEE Sens. J. 2010, 11, 1423-1426.

10. Coelho, L.; De Almeida, J.; Santos, J.L.; Viegas, D. Fiber optic hydrogen sensor based on an etched Bragg grating coated with palladium. Appl. Opt. 2015, 54, 10342-10348.

11. Dai, J.; Yang, M.; Chen, Y.; Cao, K.; Liao, H.; Zhang, P. Side-polished fiber Bragg grating hydrogen sensor with WO 3-Pd composite film as sensing materials. Opt. Express 2011, 19, 6141-6148.

12. Liu, C.; Wang, J.; Wang, F.; Su, W.; Yang, L.; Lv, J.; Fu, G.; Li, X.; Liu, Q.; Sun, T. Surface plasmon resonance (SPR) infrared sensor based on D-shape photonic crystal fibers with ITO coatings. Opt. Commun. 2020, 464, 125496.

13. Girei, S.H.; Shabaneh, A.A.; Ngee-Lim, H.; Hamidon, M.N.; Mahdi, M.A.; Yaacob, M.H. Tapered optical fiber coated with graphene based nanomaterials for measurement of ethanol concentrations in water. Opt. Rev. 2015, 22, 385-392.

14. González-Sierra, N.E.; Gómez-Pavón, L.D.C.; Pérez-Sánchez, G.F.; Luis-Ramos, A.; Zaca-Morán, P.; Muñoz-Pacheco, J.M.; Chávez-Ramírez, F. Tapered optical fiber functionalized with palladium nanoparticles by drop casting and laser radiation for $\mathrm{H} 2$ and volatile organic compounds sensing purposes. Sensors 2017, 17, 2039.

15. Wang, Z.; Xu, C.; Gao, G.; Li, X. Facile synthesis of well-dispersed Pd-graphene nanohybrids and their catalytic properties in 4-nitrophenol reduction. Rsc Adv. 2014, 4, 13644-13651.

16. González-Sierra, N.E.; Gómez-Pavón, L.D.C.; Pérez-Sánchez, G.F.; Luis-Ramos, A.; Zaca-Morán, P.; Muñoz-Pacheco, J.M.; Chávez-Ramírez, F. Tapered optical fiber functionalized with palladium nanoparticles by drop casting and laser radiation for $\mathrm{H} 2$ and volatile organic compounds sensing purposes. Sensors 2017, 17, doi:10.3390/s17092039.

17. Basu, S.; Basu, P.K. Nanocrystalline metal oxides for methane sensors: Role of noble metals. J. Sens. 2009, 2009, doi:10.1155/2009/861968.

18. Renganathan, B.; Sastikumar, D.; Srinivasan, R.; Ganesan, A.R. Nanocrystalline samarium oxide coated fiber optic gas sensor. Mater. Sci. Eng. B 2014, 186, 122-127.

Publisher's Note: MDPI stays neutral with regard to jurisdictional claims in published maps and institutional affiliations.

(C) 2020 by the authors. Submitted for possible open access publication under the terms and conditions of the Creative Commons Attribution (CC BY) license (http://creativecommons.org/licenses/by/4.0/). 